\title{
IMPACTO DE HERBICIDAS EM UMA GUILDA DE FORMIGAS PREDADORAS
}

\author{
Wedson D. Fernandes ${ }^{1}$, Márcia C. A. Cruz ${ }^{2}$, Odival Faccenda ${ }^{3}$ e Tarcisio O. Valente ${ }^{4}$
}

'Dr., Professor. Universidade Federal de Mato Grosso do Sul, Departamento de Ciências Biológicas. Caixa Postal 322. Dourados, MS 79825-070 wedson@ceud.ufms.br

${ }^{2}$ Acadêmica . Universidade Federal de Mato Grosso do Sul, Departamento de Ciências Exatas e Biológicas, Curso de Ciências Biológicas. ${ }^{3}$ Dr., Professor. Universidade Estadual de Mato Grosso do Sul. Dourados, MS 79825-070 faccenda@ceud.ufms.br

${ }^{4}$ M.Sc., Professor. Universidade Federal de Mato Grosso do Sul, Departamento de Ciências Agrárias. Caixa Postal 322. Dourados, MS 79825-070

\begin{abstract}
RESUMO
O impacto provocado pelos herbicidas alachlor, metolachlor, dimethenamid e diuron em uma guilda de formigas predadoras, foi avaliado em Dourados, MS (22 $13^{\prime} 16^{\prime \prime}$ e e $\left.54^{\circ} 48^{\prime} 20^{\prime \prime} \mathrm{W}\right)$. Duas áreas testemunhas, com e sem capina, foram utilizadas neste estudo. As formigas foram amostradas através de duas iscas de sardinha por parcela, com quatro repetições por tratamento. Solenopsis sp. foi a espécie mais abundante e freqüente em relação às cinco espécies encontradas nas iscas. Houve um decréscimo no número de formigas nas parcelas pulverizadas e nas testemunhas por um período de 40 dias, não tendo sido encontrada diferença significativa entre as parcelas com e sem herbicidas.
\end{abstract}

Palavras-chave: Solenopsis, Pheidole, plantas daninhas, controle natural, agroecossistema

\section{ABSTRACT \\ Impact of herbicides on a predator ant guild}

The effects of alachor, metolachlor, dimethenamid and diuron herbicides on a predator ant guild were evaluated in Dourados, MS ( $22^{\circ} 13^{\prime} 16^{\prime \prime} \mathrm{S}$ and $\left.54^{\circ} 48^{\prime} 20^{\prime \prime} \mathrm{W}\right)$. Two control areas, with and without weeds, were used in the study. The ants were sampled using two sardine baits per plot with four replications per treatment. Solenopsis sp. was the most abundant ant species among the five sampled species. There was a decrease in the number of ants in the treatment and control plots after 40 days. No significant differences were found between the herbicide and control plots.

Key words: Solenopsis, Pheidole, weeds, natural control, agroecosystem 
Wendson D. Fernandes et al.

\section{INTRODUÇÃO}

As formigas, devido à sua alta diversidade e abundância, exercem papel fundamental em vários ecossistemas. Elas podem agir como herbívoras ou predadoras, podem polinizar plantas, dispersar sementes, aerar o solo, além de auxiliar na ciclagem de nutrientes (Wilson, 1963; Young, 1981; Hölldobler \& Wilson, 1990). Muitos têm sido os estudos sobre a importância deste grupo de insetos em ecossistemas tropicais e subtropicais, principalmente em monoculturas onde atuam como agentes de controle natural de insetos pragas, sendo sua manutenção fundamental em programas de manejo integrado de pragas (Risch, 1981; Risch \& Carrol, 1982; Fernandes et al., 1994). Segundo Risch \& Carrol (1982) e Way \& Khoo (1992), formigas respondem rapidamente à densidade populacional da presa. Esses animais permanecem abundantes mesmo quando as presas estão escassas, através do canibalismo das formas imaturas e da utilização do alimento estocado. Várias espécies de formigas possuem hábitos de predação, podendo usar uma grande variedade de artrópodes como fonte de alimento (Fowler et al., 1991). Way \& Khoo (1992) citaram as espécies do gênero Solenopsis como importantes agentes de controle biológico nos trópicos e subtrópicos e Solenopsis invicta Buren, foi citada como predadora de ovos de Heliothis virescens (Fabr., 1781) por MacDaniel \& Sterling (1979 e 1982) e de Anthonomus grandis Boh. 1843 por Fillman \& Sterling (1985). Castro \& Queiroz (1987) verificaram que a espécie Pheidole gertrudae Forel 1886, pode ser utilizada como agente de controle biológico de insetos pragas e plantas invasoras. Fernandes et al. (1994), demonstraram na região de Campinas (SP), que aproximadamente $20 \%$ dos adultos de $A$. grandis oferecidos no solo, são atacados e removidos por formigas, sendo que a espécie mais freqüente foi Pheidole oliveirai (Wilson \& Brown, em processo de revisão taxonômica). Em observações realizadas em Dourados (MS), formigas do gênero Solenopsis foram as mais abundantes em campos de soja, sendo que três espécies do gênero Pheidole e mais Dorymyrmex também tiveram uma distribuição regular em todo o período de observação.

Apesar de sua importância como agentes de controle natural as formigas sofrem, direta ou indiretamente, uma drástica redução populacional devido ao efeito de agroquímicos que são constantemente utilizados para o controle de pragas e plantas daninhas. $\mathrm{O}$ uso de herbicidas para o controle de plantas daninhas tem sido amplamente adotado em substituição aos métodos tradicionais de capinas manuais, tração animal e tratorizada (Azevedo et al., 1988). Seu impacto sobre a fauna de artrópodos benéficos já foi estudado por diversos autores em plantações de algodão, amendoim e soja (Huckaba et al., 1983; Laster et al., 1984; Isenhour et al., 1985; Mack et al., 1987 e Soares et al., 1995). Alguns autores têm evidenciado a propriedade inseticida de alguns herbicidas, como Miller \& Miller (1979) que observaram uma redução na população de L. lineolaris após a aplicação de dinoseb, um fungicida com propriedades herbicida e insetici- da em campos algodoeiros. Segundo Stan et al. (1978), MSMA +diuron possue atividade inseticida para Frankliniella spp. e Lygus lineolaris.

O objetivo do presente estudo foi o de avaliar o impacto de herbicidas sobre a freqüência e densidade populacional em uma guilda de formigas predadoras em Dourados, MS.

\section{MATERIAL E MÉTODOS}

O experimento foi realizado no Núcleo de Ciências Agrárias da Universidade Federal de Mato Grosso do Sul em Dourados, MS (22 ${ }^{\circ} 13^{\prime} 16^{\prime \prime S}$ e $\left.54^{\circ} 48^{\prime} 20^{\prime \prime W}\right)$, no período de outubro de 1995 a janeiro de 1996. A área caracteriza-se pela diversidade de cultivos, principalmente para trabalhos de pesquisa, predominando soja, milho, hortaliças, trigo e frutíferas.

Foi utilizado um experimento fatorial com dois fatores: herbicidas com seis níveis e tempo em relação às aplicações dos herbicidas com oito níveis de observação. Os níveis do fator herbicida foram: $\mathrm{H} 1=$ alachlor (Laço CE, 2,8 kg/ha i.a.), $\mathrm{H} 2=$ metolachlor (Dual $900 \mathrm{CE}, 2,88 \mathrm{~kg} / \mathrm{ha}$ i.a.), H3= dimethenamid (Zeta $900 \mathrm{CE}, 1,12 \mathrm{~kg} / \mathrm{ha}$ i.a.), $\mathrm{H} 4=$ diuron (Karmex 2,6 kg/ha i.a.); mais 2 testemunhas: Ts $1=$ sem capina e Ts2= com capina. Os níveis do fator tempo foram: pré aplicação, 2, 9, 16, 24, 32, 59 e 90 dias após aplicação (DAA) dos herbicidas. O delineamento consistiu de 48 tratamentos: fator herbicidas $(n=6)$ com 4 repetições $x$ fator tempo $(n=8)$. Para execução do experimento foi utilizada uma área de $480 \mathrm{~m}^{2}$, subdividida em 24 parcelas de $20 \mathrm{~m}^{2}$, distribuídas aleatoriamente, para a verificação do impacto dos herbicidas. As coletas foram realizadas com o uso de duas iscas de pasta de sardinha, com aproximadamente duas gramas cada. As iscas foram colocadas no solo sobre papel filtro, em cada uma das parcelas, e retiradas aproximadamente 40 minutos após, sempre no período vespertino. As formigas coletadas foram levadas ao laboratório para identificação. Inúmeras pesquisas têm sido realizadas com a utilização deste método para capturas de formigas, devido à facilidade de utilização e por recrutar um grande número de gêneros ricos em espécies (Castro \& Queiroz, 1987; Caldas \& Moutinho, 1993).

Foram também realizadas duas avaliações visuais da eficiência de dessecação, aos 18 e 35 dias após a aplicação dos herbicidas. Em cada avaliação era atribuída uma nota que variou de 0 (com nenhum efeito do herbicida sobre a planta) a 100 (com morte total das plantas daninhas da parcela), considerando-se a percentagem de área coberta pela planta segundo metodologia de EWRC (1964). A testemunha capinada foi mantida com $100 \%$ de controle (Tabela 2 ), através de capinas realizadas a cada quinze dias aproximadamente. A pulverização dos herbicidas foi realizada com a finalidade de avaliar o impacto destes produtos sobre a mirmecofauna e não especificamente sua eficiência no controle de plantas daninhas. Os herbicidas foram aplicados com 
pulverizador costal com pressão constante, mantido por $\mathrm{CO}_{2}$, equipado com bicos TeeJet tipo leque (110:02). A área permaneceu sem qualquer plantio durante o período de avaliação. Os dados foram submetidos a análise de variância e aplicado o teste de Duncan a 5\% de significância.

\section{RESULTADOS E DISCUSSÃO}

Foram observadas seis espécies de formigas: Ectatomma quadridens (Fabricius, 1793) (Ponerinae), Solenopsis sp., Pheidole sp1, sp2 e sp3 (Myrmicinae) e Linepithema sp. (Dolichoderinae), que foi encontrada em uma única vez durante todo o período. A espécie mais abundante e com maior frequência relativa, encontrada nas iscas, foi Solenopsis sp., seguido de Pheidole sp1 e Pheidole sp2. As outras espécies do gênero Pheidole e E. quadridens foram pouco frequentes e abundantes.

A baixa complexidade estrutural observada, com poucas espécies vegetais e solo preparado em sistema de plantio convencional em todas as parcelas, contribuiu para a captura de poucas espécies. Segundo Southwood (1985) o aumento da complexidade no habitat resulta em maior diversidade da entomofauna e, consequentemente, uma maior disponibilidade de presas, além do aumento em sítios para nidificação. O declínio da complexidade estrutural do habitat tende a provocar um aumento da freqüência de interações agressivas na mirmecofauna (Carrol \& Jansen, 1973). Castro \& Queiroz(1987) observaram em uma comunidade de milho, uma alta capacidade de recrutamento e ocupação espacial por parte da espécie dominante, Pheidole gertrudae, sendo que o mesmo não ocorreu em comunidade tipo Cerrado.

A baixa riqueza vegetal na área estudada, com exclusivamente plantas daninhas, que também foram controladas, facilitou a ocupação mais extensiva de Solenopsis sp., uma espécie generalista e oportunista que nidifica basicamente no solo. O gênero Solenopsis pode ser encontrado em ambientes de clima quente, como as regiões tropicais e subtropicais da América do Sul (Way \& Khoo, 1992).

Solenopsis invicta faz parte do complexo de inimigos naturais de Helicoperva virescens, Alabama argillacea e Anthonomus grandis em monocultura de algodão (Gravena \& Pazetto, 1987; Fernandes et al., 1994 e MacDaniel e Sterling, 1982).

Observou-se uma redução na média de formigas coletadas após a utilização dos herbicidas, exceto para o diuron e para a testemunha sem capina, que apresentaram um ligeiro acréscimo populacional, para em seguida também diminuírem na (Tabela 1). $\mathrm{O}$ aumento na média de formigas para os dois níveis deve-se ao aumento populacional de Solenopsis sp., uma espécie dominante e bem adaptada às condições desse ambiente, que não sofreu, imediatamente, o efeito do herbicida. Entretanto esta população também experimentou uma queda acentuada pouco depois da pulverização. Segundo Castro et al. (1990) espécies do gênero Solenopsis, encontradas com pouca frequência em habitats com maior complexi-

Tabela 1. Média de formigas capturadas em diferentes períodos antes e após pulverização de herbicidas. Dourados, MS. $1995 / 96$.

\begin{tabular}{|c|c|c|c|c|c|c|c|c|c|c|c|c|c|}
\hline \multicolumn{14}{|c|}{ Média de formigas capturadas ${ }^{2,3,4}(\%)$} \\
\hline & \multicolumn{2}{|c|}{ Alachlor } & \multicolumn{2}{|c|}{ Metolachlor } & \multicolumn{2}{|c|}{ Dimethenamid } & \multicolumn{2}{|c|}{ Diuron } & \multicolumn{2}{|c|}{$\begin{array}{l}\text { Testemunha sem } \\
\text { capina }\end{array}$} & \multicolumn{2}{|c|}{$\begin{array}{l}\text { Testemunha com } \\
\text { capina }\end{array}$} & \multirow[b]{2}{*}{ Média $^{5}$} \\
\hline & \multicolumn{2}{|c|}{ Média \pm D.P. } & \multicolumn{2}{|c|}{ Média \pm D.P. } & \multicolumn{2}{|c|}{ Média \pm D.P. } & \multicolumn{2}{|c|}{ Média \pm D.P. } & \multicolumn{2}{|c|}{ Média \pm D.P. } & \multicolumn{2}{|c|}{ Média \pm D.P. } & \\
\hline Pré & 96,0 & \pm 1089 & 21,1 & $\pm 41,6$ & 5,5 & $\pm 11,0$ & 82,4 & $\pm 76,1$ & 23,0 & \pm 122 & 30,6 & $\pm 39,0$ & $45,6 \mathrm{a}$ \\
\hline $2 \mathrm{DAA}^{1}$ & 2,4 & \pm 47 & 13,7 & $\pm 27,5$ & 0,0 & $\pm 0,0$ & 121,7 & \pm 1632 & 84,1 & $\pm 24,5$ & 11,7 & $\pm 13,6$ & $42,1 \mathrm{ab}$ \\
\hline 9DAA & 0,0 & $\pm 0,0$ & 0,0 & $\pm 0,0$ & 0,0 & $\pm 0,0$ & 0,0 & $\pm 0,0$ & 4,8 & $\pm 9,5$ & 3,6 & $\pm 5,4$ & $2,8 \mathrm{~b}$ \\
\hline 16DAA & 0,0 & $\pm 0,0$ & 0,0 & $\pm 0,0$ & 0,0 & $\pm 0,0$ & 0,0 & $\pm 0,0$ & 0,0 & $\pm 0,0$ & 295 & $\pm 43,7$ & $0,6 \mathrm{~b}$ \\
\hline $24 \mathrm{DAA}$ & 0,0 & $\pm 0,0$ & 0,1 & \pm 02 & 26 & \pm 46 & 2,0 & $\pm 4,0$ & 32,8 & \pm 336 & 88,4 & $\pm 78,8$ & $11,2 \mathrm{ab}$ \\
\hline $32 \mathrm{DAA}$ & 9,7 & $\pm 10,5$ & 34,9 & $\pm 35,1$ & 17,1 & $\pm 20,9$ & 60,7 & $\pm 94,0$ & 52,5 & $\pm 14,4$ & 8,4 & $\pm 13,5$ & $43,9 a$ \\
\hline $59 \mathrm{DAA}$ & 59,4 & $\pm 97,1$ & 69,0 & $\pm 129,4$ & 22,1 & \pm 429 & 63,4 & $\pm 116,0$ & 120 & \pm 249 & 602 & $\pm 113,9$ & $39,0 \mathrm{a}$ \\
\hline $90 \mathrm{DAA}$ & 53,4 & \pm 472 & 20,7 & $\pm 29,8$ & 11,0 & $\pm 19,4$ & 402 & $\pm 71,8$ & 0,6 & \pm 12 & 31,9 & $\pm 63,1$ & $31,0 \mathrm{a}$ \\
\hline Média $^{6}$ & 27,6 & $\pm 37,0$ & 199 & $\pm 23,5$ & 7,3 & $\pm 8,56$ & 463 & $\pm 60,8$ & 262 & $\pm 11,9$ & 33,0 & \pm 287 & \\
\hline
\end{tabular}

'Dias após a aplicação dos herbicidas.

${ }^{2}$ Os valores da variável dependente representam a média de quatro repetições.

${ }^{3}$ Os valores foram transformados em $\sqrt{\mathrm{x}+0,5}$ para efeito do cálculo da análise de variância.

${ }^{4}$ Não houve diferença significativa para a interação entre os fatores herbicida e os períodos de observação, $\mathrm{F}=1,1604$ com $\mathrm{p}>0,05$.

${ }^{5}$ Médias seguidas pela mesma letra, não diferem significativamente pelo teste de Duncan ao nível de $5 \%$.

${ }^{6}$ Não houve diferença significativa entre os níveis do fator herbicida, $F=2,085$ com $p>0,05$.

${ }^{7}$ D.P. $=$ Desvio padrão. 
Wendson D. Fernandes et al.

dade espacial, foram observadas como dominantes em habitats sujeitos a estresse mais acentuado. $S$. invicta é considerada uma espécie oportunista e pioneira em processos de sucessão (Tschinkel \& Hess, 1999). Stan et al. (1978) constataram toxicidade de MSMA, diuron e dinoseb para Coleomigila maculata (De Geer), Eretmocerus haldemani, Orius insidiosus, Geocoris punctipes e Sycmnus louisiane.

O início da recuperação na guilda de formigas ocorreu aproximadamente 30 dias após a aplicação dos herbicidas, exceto para as testemunhas com capina e sem capina, que já apresentavam um acréscimo populacional 16 dias após o início do experimento. A interação entre os herbicidas e épocas de observação não foi significativa $(F=1,161$ com $p>0,05)$, indicando que a captura de formigas ao longo do tempo, foi semelhante para todos os níveis do fator herbicida, ou seja, os fatores agiram independentemente sobre a procura das iscas pelas formigas.

A queda nas populações de formigas e na frequência de iscas utilizadas na testemunha sem capina pode ter sido influenciada pelo tamanho das parcelas $\left(20 \mathrm{~m}^{2}\right)$, sofrendo um efeito repelente e consequentemente uma diminuição da densidade populacional das espécies encontradas. A testemunha 2 (capinada) sofreu ainda a influência das capinas realizadas durante o período de observação, no $2^{\circ}$ e $32^{\circ}$ dia após a pulverização dos herbicidas (Figura 1). O trânsito de formigas entre as parcelas foi observado em diversos momentos. Algumas vezes, formigas que saíam de aberturas de ninhos que estavam na parte periférica de uma parcela atacavam a isca de uma parcela adjacente. A diminuição da abundância e da freqüência de formigas nas parcelas tratadas com herbicidas provocou, indiretamente, uma diminuição na captura de formigas nas parcelas testemunhas. As médias de formigas capturadas no $9^{\circ}$ e $16^{\circ}$ dia após a aplicação reforçam a hipótese de migração entre as parcelas (Tabela 1). Após a aplicação dos herbicidas, parte da população presente nestas parcelas migrou para as testemunhas ou para a do diuron, que foi o menos tóxico dos herbicidas utilizados. Este herbicida, na formulação de grânulos dispersíveis em água, pertence a classe toxicológica IV, com toxidade aguda via oral com $\mathrm{DL}_{50}>7000$

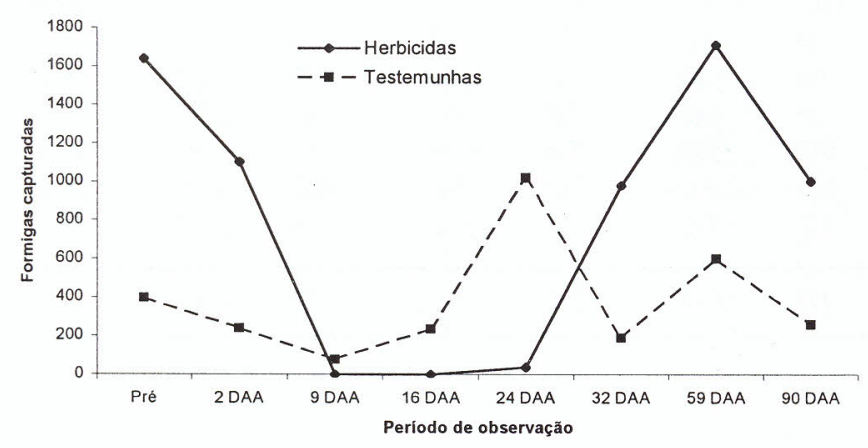

Figura 1. Total de formigas capturadas nos tratamentos com herbicidas (considerou-se os tratamentos conjuntamente) e testemunhas (considerouse as duas testemunhas conjuntamente). mg/kg para ratos. (Rodrigues \& Almeida, 1995).

Não foram observadas diferenças significativas para média de formigas coletadas entre os tratamentos com herbicidas $(\mathrm{F}=2,085$ com $\mathrm{p}>0,05$, na Tabela 1$)$, mas houve diferença significativa entre as datas de observações $(F=3,478$ com $\mathrm{p}<0,05$, na Tabela 1). De maneira geral, houve um decréscimo do número de formigas capturadas em todos os níveis do fator herbicida e nas testemunhas, após a pulverização destes, mantendo-se em baixa abundância durante aproximadamente 40 dias, quando então observou-se um aumento gradual nas populações capturadas. Nas testemunhas a queda no número total de formigas foi menor e a recuperação, em relação à quantidade inicial observada, foi mais rápida (Tabela 1 e Figura 1). O mesmo padrão foi observado quanto à percentagem de iscas ocupadas por formigas (Figura 2), embora esta percentagem nas testemunhas tenha sido, em quase todo o período, maior que as amostradas nos níveis envolvendo aplicação de diferentes herbicidas. A diferença entre os níveis do fator herbicida, quando observados conjuntamente, e entre as médias dos níveis testemunhas, também comparadas conjuntamente, foi significativa $(t=1,923 \mathrm{com}$ $\mathrm{P}<0,05)$ durante o período avaliado. Não existe entretanto diferença significativa entre a porcentagem de iscas ocupadas nas testemunhas com e sem capina $(t=1,425 \operatorname{com} P>0,05)$.

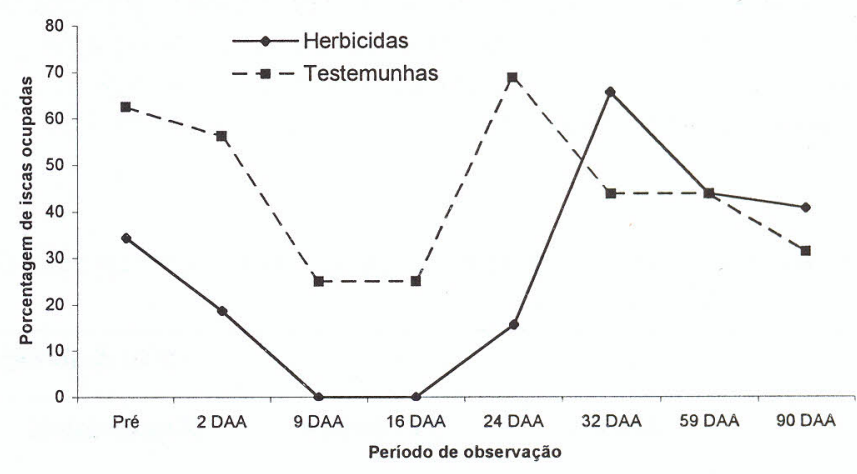

Figura 2. Percentagem de iscas ocupadas por formigas nos tratamentos com herbicidas (considerouse os tratamentos conjuntamente) e testemunhas (considerou-se as duas testemunhas conjuntamente).

Após a aplicação dos herbicidas, toda a área estudada sofreu o seu impacto, independente do tipo de produto utilizado, durante um período de aproximadamente 30 dias, recuperando logo após, médias de captura semelhantes ao período de pré-aplicação. Os resultados obtidos mostraram que os herbicidas, de uma maneira geral, exerceram impacto sobre a guilda de formigas estudadas. A toxicidade dos produtos utilizados exerceu, em um primeiro momento, um efeito relativamente drástico nas populações de formigas. Estas populações continuaram a sofrer pressões negativas durante grande parte do período, que devido ao efeito da ausência de vegetação na área, ficavam expostas à intensa radiação solar, chuva e vento durante o período de estudo, dificultando a 
movimentação e consequentemente reduzindo o número de indivíduos à procura de alimento, sendo estes fatores preponderantes para a redução na movimentação das formigas sobre o solo.

Além disso, as formigas provavelmente sofreram também efeito repelente provocado pelos herbicidas, que as afastou das parcelas tratadas, ou mesmo manteve-as no ninho durante o período de maior efeito dos herbicidas. Pela proximidade das parcelas, acredita-se que as testemunhas também tenham sido influenciadas pelo efeito dos herbicidas. Como a área testemunha $(\mathrm{T} 1+\mathrm{T} 2)$ representava $20 \%$ do total estudado, e todo o restante da área sofreu alterações, é provável que também tenha ocorrido aí alguma interferência.

Segundo Eijsackers \& Van der Drift (1976), herbicidas que normalmente são utilizados na forma de "sprays" líquidos colocam sob risco considerável populações da macrofauna do solo, como aranhas, besouros e formigas, que freqüentemente sofrem ação repelente.

A queda generalizada da densidade de formigas, no último dia de observação (90 DAA), em fevereiro, deve-se principalmente ao preparo do solo para posterior plantio (Tabela 1). Soares et al. (1995), estudando a influência de herbicidas em artrópodes relacionados à cultura algodoeira, verificaram que estes exerceram uma supressão sobre algumas espécies de predadores, principalmente Cycloneda sanguinea, Doru lineare e Scymnus sp.

As condições normais de utilização do solo, com plantio após a utilização de herbicidas, podem representar um fator benéfico para a recolonização das formigas. $\mathrm{O}$ aumento da complexidade da estrutura vegetal, com a presença de plantas semeadas, certamente irá favorecer o desenvolvimento de algumas das espécies não dominantes neste ambiente, aumentando consequentemente a riqueza de espécies no local. A utilização de plantio convencional nos anos anteriores e a ausência de plantas na área, foi um dos fatores limitantes para a guilda de formigas. A ausência da biomassa aérea de vegetação e de solo não disponibilizou uma quantidade adequada de sítios de nidificação para as espécies. Provavelmente, em áreas com sistema de plantio direto e consequentemente a manutenção de uma liteira mais rica, ocorreria um impacto menor na diversidade e abundância de espécies. A complexidade estrutural desta liteira com maior disponibilidade de nichos, proporcionaria uma maior quantidade de refúgios possi- bilitando um menor impacto dos herbicidas sobre as populações.

O impacto global de formigas em agroecossistemas pode ser benéfico, promovendo um efetivo controle biológico de pragas (Risch \& Carrol, 1982), sendo que a manutenção de toda a fauna benéfica, incluindo as formigas, representa um componente importante para este controle, favorecendo a diminuição na quantidade de inseticidas utilizados e, consequentemente, reduzindo os problemas observados em relação aos resíduos tóxicos, resistência, surgimento de pragas secundárias e ressurgência. O conhecimento das interações multitróficas no ambiente é um dos componentes para a construção de um manejo sustentável do ambiente (Lewis et al., 1997).

Em relação às plantas daninhas, houve uma dominância quase total de capim marmelada (Brachiaria plantaginea Link) e um bom nível de controle com todos os herbicidas (Tabela 2).

Estudos complementares para análise qualitativa e quantitativa do potencial de formigas como agentes de controle natural, poderão fornecer informações valiosas para a utilização destas espécies em programas de manejo integrado de pragas em agroecossistemas.

\section{CONCLUSÕES}

Não foram observadas diferenças significativas para o efeito dos herbicidas alachlor, metolachlor, dimethenamid e diuron. Após a aplicação dos herbicidas, toda a área estudada sofreu o seu impacto, independente do tipo de produto utilizado, durante um período de aproximadamente 30 dias, recuperando logo após, as médias de captura do período de préaplicação.

\section{LITERATURA CITADA}

AZEVEDO, D. M. P.; VIEIRA, D. J.; NÓBREGA, L. D.; BELTRÃO, N. E. M. Controle de ervas daninhas e seletividade de herbicidas em algodoeiro herbáceo. Pesq. Agropec. Brasil., v. 23, p. 581-586, 1988.

Tabela 2. Nível de controle de plantas daninhas, em percentagem, exercido pelos diferentes tratamentos com herbicidas e nas testemunhas. Dourados, MS. 1995/96.

\begin{tabular}{ccccccc}
\hline \multirow{2}{*}{$\begin{array}{c}\text { DIAS APÓS } \\
\text { A } \\
\text { APLICAÇÃOO }\end{array}$} & Alachlor & Metolachlor & Dimethenamid & Diuron & $\begin{array}{c}\text { Testemunhasem } \\
\text { capina }\end{array}$ & $\begin{array}{c}\text { Testemunha com } \\
\text { capina }\end{array}$ \\
\cline { 2 - 7 } & Média \pm D.P & Média \pm D.P. & Média \pm D.P. & Média \pm D.P. & Média \pm D.P. & Média \pm D.P. \\
\hline 18 & $86 \pm 3,0$ & $75 \pm 4.1$ & $86 \pm 4,3$ & $91 \pm 3,0$ & 0 & 100 \\
35 & $81 \pm 6,7$ & $71 \pm 10,3$ & $86 \pm 4,8$ & $82 \pm 18,2$ & 0 & 100 \\
\hline
\end{tabular}

${ }^{1}$ D.P.: Desvio padrão 
Wendson D. Fernandes et al.

CALDAS, A.; MOUTINHO, P.R.S. Composição e diversidade da fauna de formigas (Hymenoptera, Formicidae) em áreas sob remoção experimental de ávores na reserva flo res-tal de Linhares, ES, Brasil. Rev. Bras. Ent., v. 37, p. 299-304, 1993.

CARROL, C.R.; JANSEN, D.H. Ecology of foraging by ants. Ann. Rev. Ecol. Syst., v. 4, p. 231-257, 1973.

CASTRO, A.G.; QUEIROZ, M.V.B. Estrutura de uma comunidade de formigas em agro-ecossistema neotropica. An. Soc. Entomol. Brasil, v.16, p.363-375, 1987.

CASTRO, A.G., QUEIROZ, M.V.B.; ARAÚJO, L.M. O papel do distúrbio na estrutura de comunidades de formigas (Hymenoptera, Formicidae). Rev. Bras. Ent., v. 34, p. 201213, 1990.

EIJSACKERS, H.; VAN DER DRIFT, J. Effects on the soil fauna. In: L.J. Audus (ed.). Herbicides. Vol. 2, $2^{\text {nd }}$ ed., 564 p. $1976 .$, p. $149-174$

EUROPEAN WEED RESEARCH COUNCIL-EWRC. Methods in weed research. Weed Research, Oxford, v.4, p. 88.1964.

FERNANDES, W.D.; OLIVEIRA, P.S.; CARVALHO, S.L.; HABIB, M.E.M.. Pheidole ants as potential biological control agents of the boll weevil, Anthonomus grandis (Coleoptera: Curculionidae), in Southest Brazil. J. Appl. Ecol., v.18, n.4/5, p. 437-441, 1994.

FILLMAN, D. A.; STERLING, W. L. Inaction level for the red imported fire ant, Solenopsis invicta (Hym: Formicidae): a predator of the boll weevil, Anthonomus grandis (Col.:Curculionidae). Agric. Ecossyst. Environ., v.13, p. 93-1102, 1985.

FOWLER, H. G.; FORTI, L.C.; BRANDÃO, C.R.F.; DELABIE, J.H.C.; VASCONCELOS, H.L. Ecologia nutricional de formigas. In: Panizzi, A.R.; J.R.P. Parra (eds.). Ecologia nutricional de insetos e suas implicações no manejo de pragas. São Paulo: Manole, 359 p. 1991. p. 131-223.

GRAVENA, S.; PAZETTO, J.A.. Predation and parasitism of cotton leafworm eggs, Alabama argillacea (Lep. : Noctuidae). Entomophaga, v. 32, p. 241-248, 1987.

HÖLLDOBLER, B.; WILSON, E. O. The ants. Cam bridge, Massachusetts: Harvard University Press, 1990.

HUCKABA, R.M.; BRADLEY JR., J.R.; VANDUYN, J.W. Effects of herbicidal applications of toxaphene on the soybean thrips, certain predators, and car larworm in soybeans. J. Georgia Entomol. Soc., v. 18, p. 200-207, 1983.
ISENHOUR, D.J.; TODD, J.W.; HAUSER, E.W. The impact of toxaphene applied as a postemergence herbicide for control of sicklepod, Cassia obtusifolia L., on arthropods associated with soybean. Crop Protection, v.4, p. 434-445, 1985.

LASTER, M.L.; BAKER, R.S.; KITTEN, W.F. Effects of dinoseb and dinoseb + MSMA on arthropod populations in cotton fields. J. Econ. Entomol., v. 77, p. 741$743,1984$.

LEWIS, W.J.; VAN LENTEREN, J.C.; PHATAK, S.C.; TUMLINSON, J.H. A total system approach to sustain able pest management. Proc. Natl. Acad. Sci., v. 94, p. 12243-12248, 1997.

MACDANIEL, S.G.; STERLING, W.L. Predator determination and efficiency on Heliothis virescens eggs on cotton using ${ }^{32}$ P . Environ. Entomol., v. 8, p. 1083-1087, 1979.

MACDANIEL, S.G.; STERLING, W.L. Predation of Heliothis virescens (F.) eggs on cotton in Texas. Environ. Entomol., v. 11, p. 60-63, 1982.

MACK, T.P.; WALKER, R.H.; WEHTJE, G. Impact of sicklepod control on several insect pests and their arthropod natural enemies in florunner peanuts. Crop Protection, v. 6, p. 185-190, 1987.

MILLER, W.O.; MILLER, C.E. Plant bug reduction through the use of Premerge 3 dinitro amine herbicide as a directed spray in cotton. Down To Earth, Midland, v. 35, p.14-15, 1979.

RISCH, S. J. Ants as important predators of rootworm eggs in the neotropic. J. Econ. Entomol., v. 74, p. 88-90, 1981.

RISCH, S. J.; CARROLL, C. R., The ecological role of ants in two mexican agroecosystems. Oecologia, v. 55, p. 114 $119,1982$.

RODRIGUES, B. N.; ALMEIDA, F. S. Guia de herbicidas. 4 ed. Londrina: Ed. dos Autores, 1998.675 p.

SOARES, J.J.; BRAZ, B.A.; BUSOLI, A.C. Impacto de herbicidas sobre artrópodos benéficos associados ao algodoeiro. Pesq. Agropec. Bras., v. 30, p. 1135-1140, 1995.

SOUTHWOOD, T.R.E. Interactions of plants and animals: patterns and processes. Oikos, v. 44, p. 5-11, 1985.

STAN, P.A.; CLOWER, D.F.; GRAVES, J.B.; SCHILLING, P.E. Effects of certain herbicides on some insects and spiders found in Louisiana cotton fields. J. Econ. Entomol., v. 71, p. 477-480, 1978. 
Impacto de Herbicidas...

TSCHINKEL, W.R.; HESS, C.A. Arboreal ant community of a pine forest in Northern, Florida. Ann. Entomol. Soc. Am., v. 92, p. 63-70, 1999.

WAY, M.J.; KHOO, K.C. Role of ants in pest management. Ann. Rev. Entomol., v. 37, p. 479-503, 1992.

WILSON, E.O. The social biology of ants. Ann. Rev. Entomol., v. 8, p. 345-368, 1963.

YOUNG, A.M. Giant neotropical ant Parapnera clavata visits
Heliconia pogonantha bracts in premontane tropical rain forest. Biotropica, v. 13, p. 233, 1981.

\section{AGRADECIMENTOS}

Ao Prof. Dr. Paulo Sérgio Oliveira (Dept ${ }^{\circ}$ de Zoologia/ UNICAMP, Campinas, SP) e Inara Roberta Leal pela identificação das formigas. Aos Profs. Drs. Frederico Lopes Santos (UFMS), Honório Roberto dos Santos (UFMS), Valter V. Alves Júnior (UFMS), Kleber Del Claro (UFU) e Rogério Parentoni (UFMG), pela revisão e sugestões ao manuscrito. 
